\title{
A New Broadband MIMO Antenna System for Sub 6 GHz 5G Cellular Communications
}

\author{
Naser Ojaroudi Parchin ${ }^{* 1}$, Yasir I. A. Al-Yasir ${ }^{1}$, Ahmed M. Abdulkhaleq ${ }^{1}$, Haleh Jahanbakhsh Basherlou ${ }^{2}$, Atta \\ Ullah $^{1}$, and Raed A. Abd-Alhameed ${ }^{1}$ \\ ${ }^{1}$ Faculty of Engineering and Informatics, University of Bradford, Bradford BD7 1DP, UK \\ ${ }^{2}$ Bradford College, Bradford, West Yorkshire, BD7 1AY, UK \\ *N.OjaroudiParchin@Bradford.ac.uk
}

\begin{abstract}
A new MIMO antenna system with broadband antenna radiators is introduced for sub $6 \mathrm{GHz}$ fifth-generation (5G) mobile communications. The proposed design contains four pairs of compact coplanar-waveguide (CPW)-fed antennas with polarization diversity that are symmetrically placed at four corners of the smartphone printed circuit board (PCB). Therefore, the proposed 5G antenna design contains four horizontally polarized and four vertically-polarized antenna elements in total. A low-cost FR-4 substrate $\left(\varepsilon_{r}=4.4, \delta=0.02\right)$ with a dimension of $75 \times 150 \mathrm{~mm}^{2}$ is employed as the mainboard substrate. The proposed design offers good isolation, dual-polarized full radiation coverage, and sufficient efficiencies. In addition, a wide impedance bandwidth ( $\left.\mathrm{S}_{11} \leq-10 \mathrm{~dB}\right)$ of 3.3 to $6 \mathrm{GHz}$ has been obtained for each antenna radiator. Moreover, the proposed design exhibits sufficient performance in the presence of the human-hand.
\end{abstract}

Keywords -5G, CPW-fed antenna, polarization diversity, smartphone antenna.

\section{INTRODUCTION}

MIMO technology is probably the most promising technology to reach the required transfer data rates of fifthgeneration $(5 \mathrm{G})$ cellular communications [1-2]. It can enhance channel capacity and link reliability of the wireless system [3]. A MIMO mobile-phone system needs a number of antenna elements which operate concurrently to achieve system diversity gain [4]. Among various MIMO antennas, microstrip antenna elements are more applicable to be used in handheld devices due to their compact profile, low cost, easy integration, and manufacturability [5-8]. To realize MIMO operation for smartphones, the multi-antenna design can be used in different corners of PCB having polarization and pattern diversity for reliable communication with minimum mutual coupling [9-10].

Recently, several MIMO antenna designs have been introduced for sub $6 \mathrm{GHz} 5 \mathrm{G}$ cellular systems [11-17]. However, these smartphone antennas either exhibit narrow impedancebandwidth, use single-fed/single-polarized radiators, or employ uniplanar structures occupying large spaces of the PCB lead to increase the system complexity. We presented here a new $5 \mathrm{G}$ smartphone antenna with four-element/eight-port radiators with CPW feeding technique providing wide impedance bandwidth and polarization/pattern diversity function.
The CPW-fed antennas are widely used and becoming increasingly popular in wireless applications owing to their attractive features such as compact-size, conformal, light in weight, easy to fabricate and integrate with wireless systems. Furthermore, compared with probe-fed and microstrip-fed antennas, CPW-fed antenna can easily achieve the wideband impedance matching [18-20].

The proposed MIMO antenna design consists of four pairs of compact dual-polarized antennas deployed at the four corners of the mainboard. It is implemented on a cheap FR-4 substrate. Each antenna element contains a trapezoid-shaped slot resonator with an L-shaped stub. The arrow-shaped strip between the adjacent elements can act as a decoupling structure to improve the isolation of the slot antennas. The elements are compact in size and operating in the frequency range of 3.35-6 $\mathrm{GHz}$. The design not only generates dual-polarization function but also provides the required full radiation coverage. Properties of the CPW-fed slot antenna element and the presented MIMO smartphone design are obtained using CST software [21].

\section{Single-Element/DuAL-Polarized ANTENNA}

The characteristics of the single-element antenna have been discussed in this section. Figure 1 depicts the schematic of the antenna. It is composed of two closely-spaced trapezoidal slot resonators with L-shaped radiation stubs.

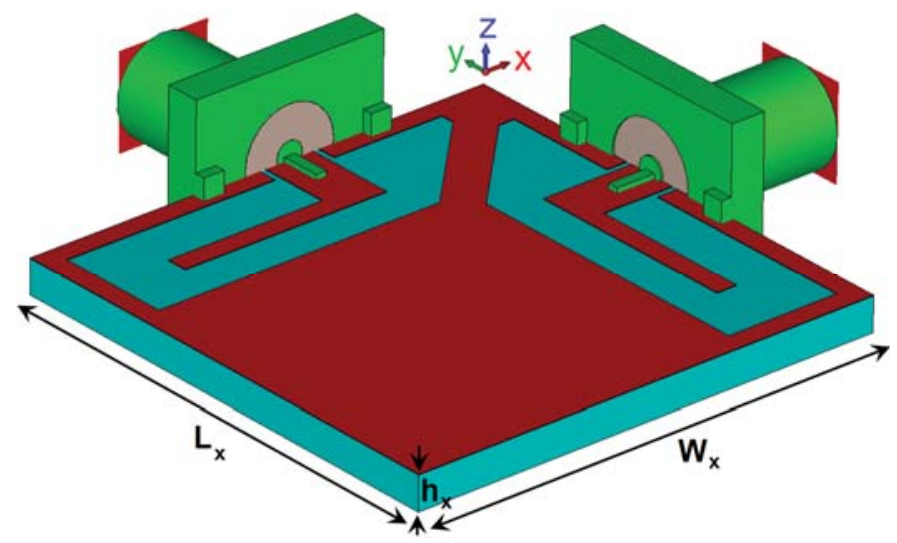

Fig. 1. Configuration of the CPW-fed dual-polarized antenna. 
Figure 2 shows the antenna S-parameters. It is clear that for $\mathrm{S}_{\mathrm{nn}} \leq-10 \mathrm{~dB}$, the antenna provides a broad impedance bandwidth of 3.2-6 GHz. In addition, a high isolation characteristic has been achieved for the designed CPW-fed antenna, especially at the center frequency.

3D Radiation patterns for different feeding ports are provided in Fig. 3. It can be seen that the designed antenna provides a good dual-polarization characteristic with a $90^{\circ}$ difference and similar radiation pattern performances with $3 \mathrm{~dB}$ IEEE gain. In addition, as clearly seen, the radiation patterns of the proposed antenna can cover both top/bottom sides of the substrate. This makes it more suitable for smartphone antenna since it can increase the radiation coverage. This has been achieved due to the nature of the employed slot radiators which can provide broadside radiation mode [22].



Fig. 2. S-parameters of the design CPW-fed antenna.

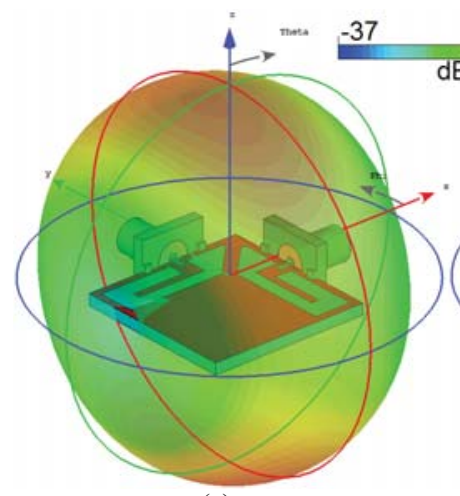

(a)

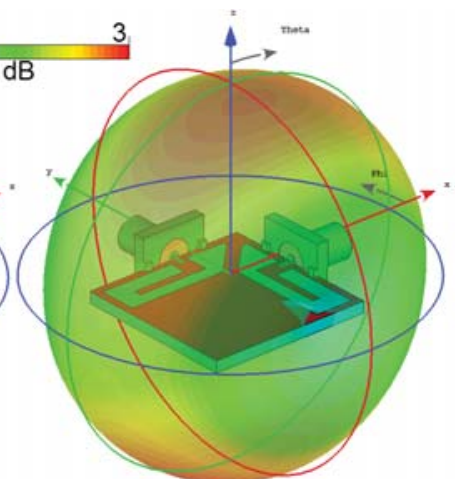

(b)
Fig. 3. 3D radiation patterns of CPW-fed design for (a) Port.1 and (b) Port. 2.

Figure 4 illustrates the fundamental radiation characteristics of the dual-polarized antenna including radiation efficiency (R.E.), total efficiency (T.E.), and maximum gain (M.G.). As can be observed, the antenna provides more than $80 \%$ efficiencies over the frequency range of 3.2 to $6 \mathrm{GHz}$. In addition, the maximum gain of the CPW-fed design varies from 2.5 to $3.5 \mathrm{dBi}$ over the operation band of the antenna.

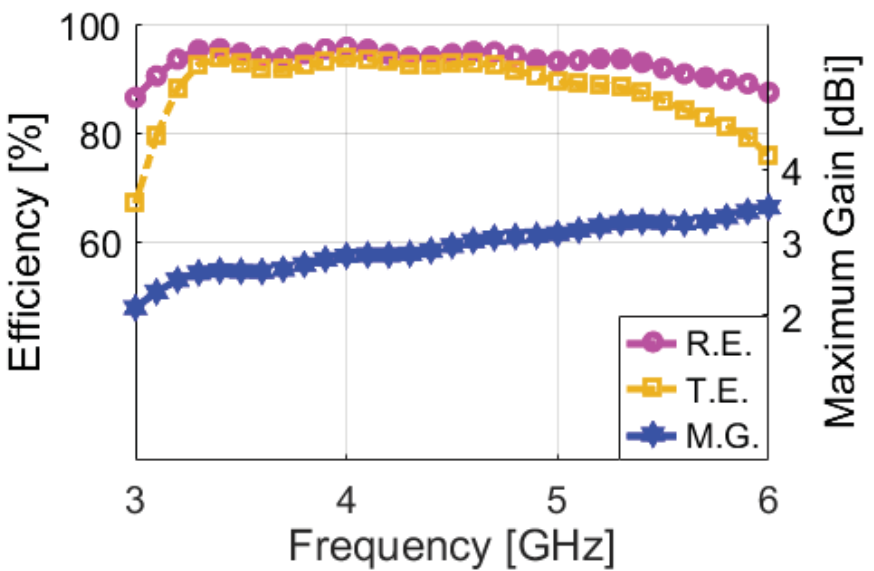

Fig. 4. Efficiencies and maximum gain of the antenna.

\section{CHARACTERISTICS OF THE SMARTPHONE ANTENNA}

Figure 5 illustrates the schematic of the presented MIMO antenna design for future smartphones. The design has been implemented on an FR4 dielectric $\left(\varepsilon_{r}=4.4\right.$ and $\left.\delta=0.02\right)$ with the size of $75 \times 150 \times 1.6 \mathrm{~mm}^{3}$. As illustrated, four pairs of the dualpolarized CPW-fed radiators have been placed at the corner of the mainboard. Each pair of microstrip lines will excite orthogonal polarizations to enhance the MIMO performances of the design [23].

Parameter values for the proposed 5G smartphone antenna array and its single-element radiator are listed in Table I. Figure 6 illustrates the $\mathrm{S}$ parameters (including $\mathrm{S}_{\mathrm{nn}}$ and $\mathrm{S}_{\mathrm{mn}}$ ) of the designed smartphone antenna. As illustrated, the antenna exhibits good $\mathrm{S}$ parameters with more than $3.5 \mathrm{GHz}$ impedance bandwidth and acceptable isolations better than $-10 \mathrm{~dB}$.

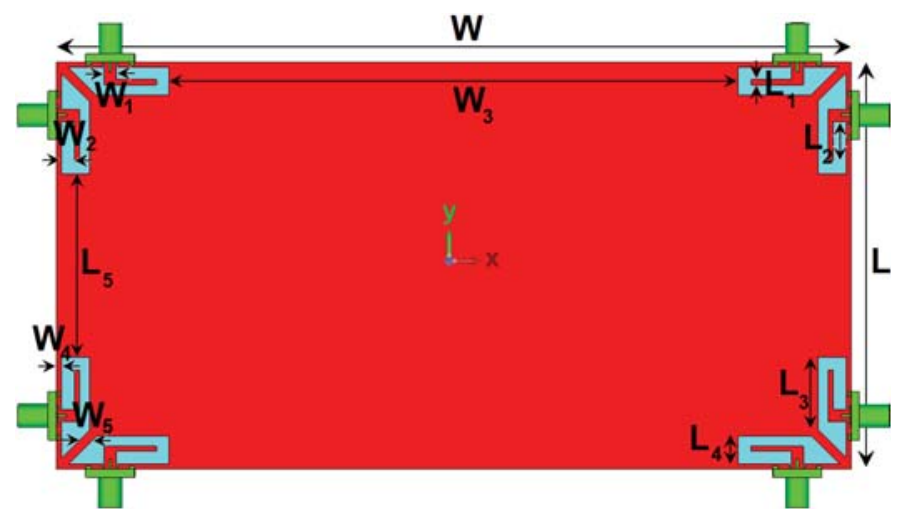

Fig. 5. Schematic of the broadband dual-polarized smartphone antenna.

TABLE I. DIMENSIONS OF THE ANTENNA PARAMETERS

\begin{tabular}{|c|c|c|c|c|c|c|c|}
\hline Parameter & $\mathrm{W}_{\mathrm{X}}$ & $\mathrm{L}_{\mathrm{X}}$ & $\mathrm{W}$ & $\mathrm{L}$ & $\mathrm{W}_{1}$ & $\mathrm{~L}_{1}$ & $\mathrm{~W}_{2}$ \\
\hline Value $(\mathrm{mm})$ & 23 & 23 & 150 & 75 & 2.4 & 1 & 2.5 \\
\hline Parameter & $\mathrm{L}_{2}$ & $\mathrm{~W}_{3}$ & $\mathrm{~L}_{3}$ & $\mathrm{~W}_{4}$ & $\mathrm{~L}_{4}$ & $\mathrm{~W}_{5}$ & $\mathrm{~L}_{5}$ \\
\hline Value $(\mathrm{mm})$ & 7.8 & 111 & 14.17 & 1 & 5.5 & 1.8 & 36 \\
\hline
\end{tabular}


It should be noted that the lower frequency of the design is shifted from 3.2 (for single-element) to $3.35 \mathrm{GHz}$. Furthermore, as shown, the mutual coupling of the closely-spaced has been decreased. These are mainly due to the MIMO configuration and large-size ground plane of the mainboard.

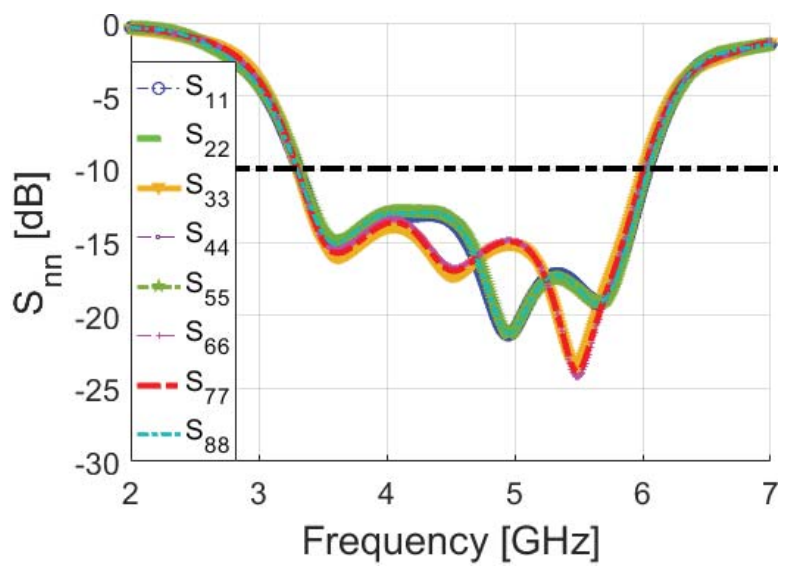

(a)

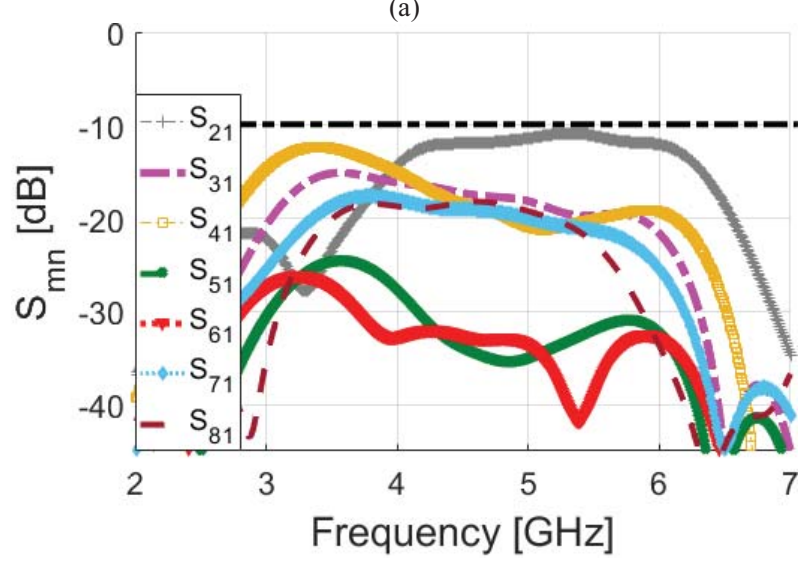

(b)

Fig. 6. (a) $\mathrm{S}_{\mathrm{nn}}$ and (b) $\mathrm{S}_{\mathrm{mn}}$ results of the designed smartphone antenna.

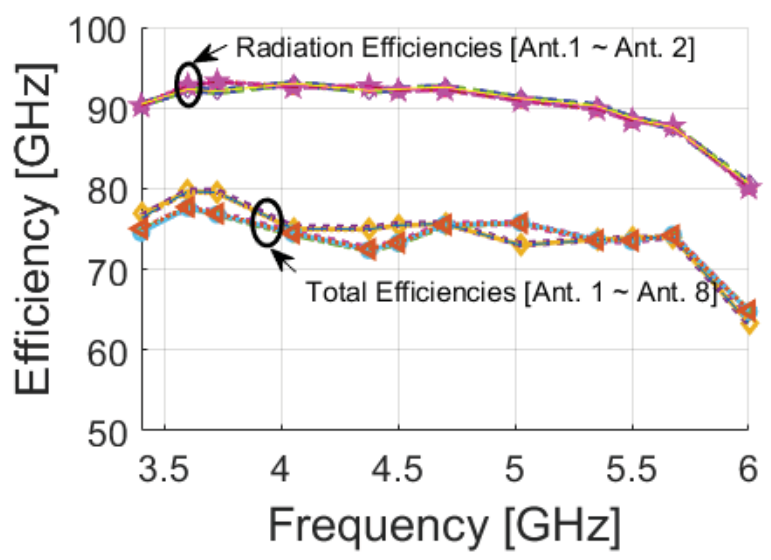

Fig. 7. Efficiencies of the antenna radiators.

The antenna elements of the designed MIMO smartphone antenna provide good radiation and total efficiencies over the operation band, as shown in Fig. 7: more than $80 \%$ radiation efficiency and $70 \%$ total efficiency properties are achieved for the elements in the wide range of 3.4-6 GHz.

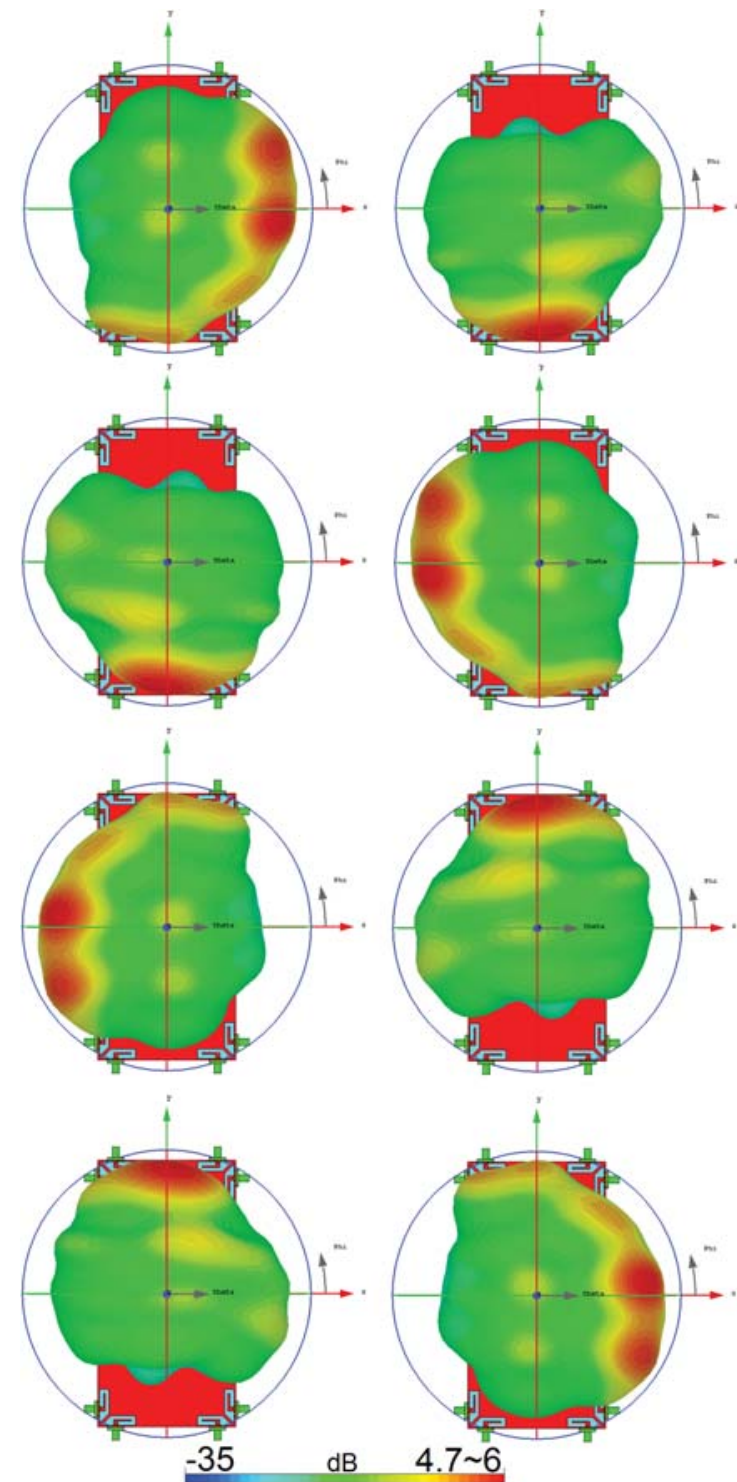

Fig. 8. 3D radiation patterns of the elements at $4.5 \mathrm{GHz}$.

The $3 \mathrm{D}$ radiation patterns for the slot radiators of the main design are displayed in Fig. 8. As shown, the 8-element MIMO antenna can cover each side of the mainboard with high-gain radiation patterns. In addition, due to the dual-polarization function of the antenna pairs, four horizontally polarized and four vertically-polarized radiation patterns are achieved to improve the MIMO performance of the design.

In the following, efficiency characteristics of the design in the presence of the human-hand in data-mode are represented. As shown different scenarios of the user-hand including righthand/left-hand modes for different design layers are investigated in Fig. 9. Due to the symmetric schematic of the proposed design, the MIMO antenna system performs almost similarly for the investigated hand scenarios. As can be observed the MIMO design and its radiation elements provide sufficient efficiencies $(40 \%-65 \%)$. The maximum reductions of the antenna efficiencies are achieved for the antenna radiators partially covered by the human-hand [24-25]. 

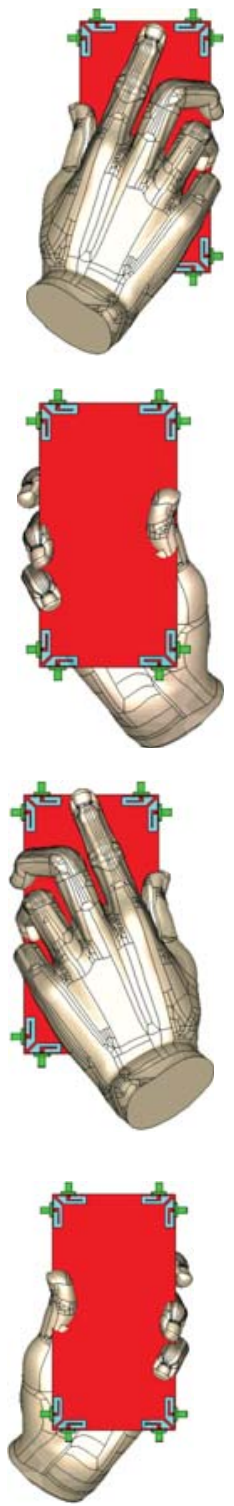

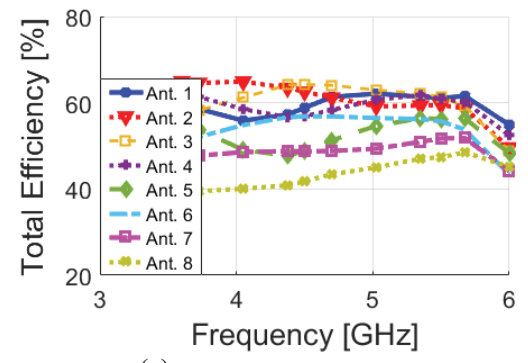

(a)

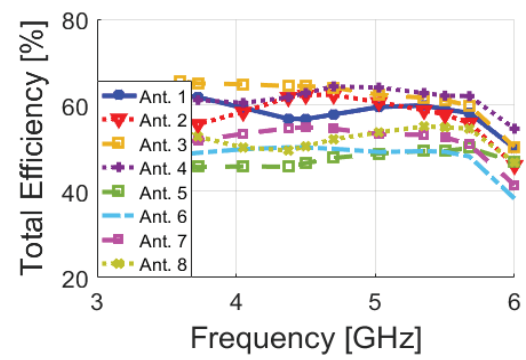

(b)

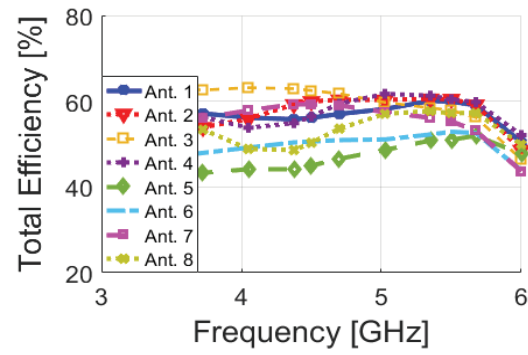

(c)

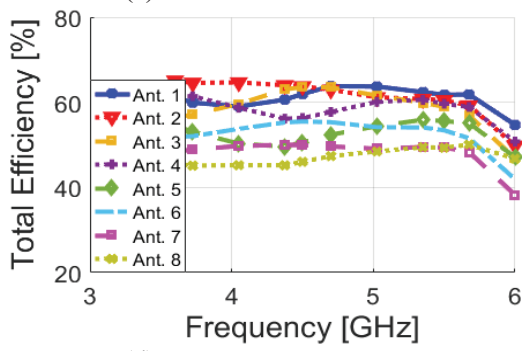

(d)

Fig. 9. Placement and the obtained efficiencies of the design for the studied hand scenarios, (a) right-hand in top-layer, (b) right-hand in back-layer, (c) lefthand in top-layer, and (d) left-hand in back-layer.

\section{CONCLUSION}

A new mobile-phone antenna design with CPW-fed antenna elements is proposed in this manuscript. The design configuration consists of dual-polarized CPW-fed slot elements deployed at the four corners of the smartphone mainboard. The antenna is designed to cover a broad frequency range of 3.3-6 GHz. The proposed MIMO antenna not only can offer wide impedance bandwidth but also supports dual polarizations. Moreover, different user-hand scenarios are investigated.

\section{ACKNOWLEDGMENT}

This work is supported by the European Union's Horizon 2020 research and innovation programme under grant agreement H2020-MSCA-ITN-2016 SECRET-722424.

\section{REFERENCES}

[1] M. Jensen, J. Wallace, “A review of antennas and propagation for MIMO wireless communications", IEEE Trans. Antennas Propag., vol. 52 2810-2824, 2004.

[2] J. Mazloum, et al., "Compact triple-band S-shaped monopole diversity antenna for MIMO applications,” ACES Journal, vol. 28, pp. 975-980, 2015.

[3] Q.U.A. Nadeem, et al., "Design of 5G full dimension massive MIMO systems," IEEE Trans. Commun., vol. 66, pp. 726-740, 2018.

[4] H.H. Yang,; Y.Q.S. Quel, "Massive MIMO Meet Small Cell. SpringerBriefs in Electrical and Computer Engineering, 2017. DOI 10.1007/978-3-319-43715-6_2.

[5] M. S. Sharawi, "Printed MIM $\bar{M}$ antenna engineering," 2014, Norwood, MA, USA: Artech House.

[6] N. Ojaroudi, "Design of microstrip antenna for 2.4/5.8 GHz RFID applications," German Microwave Conference, Aachen University, Germany, March 10-12, 2014.

[7] A. Ullah, N. O. Parchin, et al., "Coplanar waveguide antenna with defected ground structure for $5 \mathrm{G}$ millimeter wave communications," IEEE MENACOMM'19, Bahrain, 2019.

[8] N. Ojaroudi, N. Ghadimi, "Design of CPW-Fed slot antenna for MIMO system applications," Microwave Opt Technol Lett vol. 56, pp.1278$1281,2014$.

[9] N. O. Parchin, et al., " $8 \times 8$ MIMO antenna system with coupled-fed elements for 5G handsets," The IET Conference on Antennas and Propagation (APC), 11-12 November, 2019, Birmingham, UK.

[10] N. Ojaroudiparchin, et al., "A compact design of planar array antenna with fractal elements for future generation applications", ACES Journal, pp. 789-796, 2016.

[11] M.-Y. Li, et al., "Tri-polarized 12-antenna MIMO array for future 5G smartphone applications," IEEE Access, vol. 6, pp. 6160-6170, 2018.

[12] A. Zhao, R. Zhouyou, "Size reduction of self-isolated MIMO antenna system for $5 \mathrm{G}$ mobile phone applications," IEEE Antennas and Wireless Propagation Letters, vol. 18, pp. 152-156, 2019.

[13] N. O. Parchin, et al., "Eight-element dual-polarized MIMO slot antenna system for 5G smartphone applications," IEEE Access, vol. 9, pp.15612$15622,2019$.

[14] M. Abdullah, et al., "Eight-element antenna array at 3.5 GHz for MIMO wireless application," PIER C, vol. 78, pp. 209-217, 2017.

[15] N. O. Parchin, et al., "Dual-polarized MIMO antenna array design using miniaturized self-complementary structures for $5 \mathrm{G}$ smartphone applications," EuCAP Conference, April 2019, Krakow, Poland.

[16] Y. Li, et al., "High-isolation 3.5-GHz 8-antenna MIMO array using balanced open slot antenna element for 5G smartphones," IEEE Trans. Antennas Propag., 2019, doi:10.1109/TAP.2019.2902751.

[17] N. O. Parchin et al., "Frequency reconfigurable antenna array with compact end-fire radiators for 4G/5G mobile handsets," IEEE 2nd $5 G$ World Forum (5GWF), Dresden, Germany, 2019.

[18] A. Valizade, et al., "CPW-fed small slot antenna with reconfigurable circular polarization and impedance bandwidth characteristics for DCS/WIMAX applications," Prog. Electromagn. Res. C, vol. 56, pp. 65-72, 2015.

[19] A. Kamalvand, et al., "Omnidirectional/multi-resonance CPW-fed small slot antenna for UWB applications," ACES Journal, vol. 28, pp. 829$835,2013$.

[20] N. Ojaroudi and N. Ghadimi, "Dual-band CPW-fed slot antenna for LTE and WiBro applications," Microw. Opt. Technol. Lett., vol. 56, pp. 1013$1015,2014$.

[21] CST Microwave Studio, ver. 2017, CST, Framingham, MA, USA, 2017.

[22] A. Musavand, et al., "A compact UWB slot antenna with reconfigurable band-notched function for multimode applications," ACES Journal, vol. 31, pp. 14-18, 2016.

[23] N. O. Parchin et al., "Mobile-phone antenna array with diamond-ring slot elements for $5 \mathrm{G}$ massive MIMO systems," Electronics, vol. 8, pp. $521,2019$.

[24] I. Syrytsin et al., "Performance investigation of a mobile terminal phased array with user effects at $3.5 \mathrm{GHz}$ for LTE advanced," IEEE Antennas Wirel. Propag. Lett., vol. 16, pp. 1847-1850, 2017.

[25] N. Ojaroudiparchin, et al., "Design of Vivaldi antenna array with endfire beam steering function for $5 \mathrm{G}$ mobile terminals", $23 \mathrm{rd}$ Telecommunications Forum, Belgrade, Serbia, pp. 587-590, 2015. 